\title{
Scanning Tunneling Spectroscopy Study of the Proximity Effect in a Disordered Two-Dimensional Metal
}

\author{
L. Serrier-Garcia, ${ }^{1}$ J. C. Cuevas, ${ }^{2}$ T. Cren,,${ }^{1, *}$ C. Brun, ${ }^{1}$ V. Cherkez,${ }^{1}$ F. Debontridder,${ }^{1}$ \\ D. Fokin, ${ }^{1,3}$ F. S. Bergeret, ${ }^{4}$ and D. Roditchev ${ }^{1}$ \\ ${ }^{1}$ Institut des Nanosciences de Paris, Université Pierre et Marie Curie (UPMC) and CNRS-UMR 7588, \\ 4 place Jussieu, 75252 Paris, France \\ ${ }^{2}$ Departamento de Física Teórica de la Materia Condensada, Universidad Autónoma de Madrid, E-28049 Madrid, Spain \\ ${ }^{3}$ Joint Institute for High Temperatures, Russian Academy of Sciences, 125412 Moscow, Russia \\ ${ }^{4}$ Centro de Física de Materiales (CFM-MPC), Centro Mixto CSIC-UPV/EHU and Donostia International Physics Center (DIPC), \\ Paseo Manuel de Lardizabal 4, E-20018 San Sebastián, Spain
}

(Received 14 January 2013; published 9 April 2013)

\begin{abstract}
The proximity effect between a superconductor and a highly diffusive two-dimensional metal is revealed in a scanning tunneling spectroscopy experiment. The in situ elaborated samples consist of superconducting single crystalline $\mathrm{Pb}$ islands interconnected by a nonsuperconducting atomically thin disordered $\mathrm{Pb}$ wetting layer. In the vicinity of each superconducting island the wetting layer acquires specific tunneling characteristics which reflect the interplay between the proximity-induced superconductivity and the inherent electron correlations of this ultimate diffusive two-dimensional metal. The observed spatial evolution of the tunneling spectra is accounted for theoretically by combining the Usadel equations with the theory of dynamical Coulomb blockade; the relevant length and energy scales are extracted and found in agreement with available experimental data.
\end{abstract}

PACS numbers: 74.45.+c, 74.55.+v, 74.78.Na

When a normal metal $(N)$ is in close proximity to a superconductor $(S)$, it acquires genuine superconducting properties which are reflected in the modification of its local density of states (DOS). This phenomenon, known as the proximity effect, has been studied for decades [1] and is used nowadays in various superconducting quantum devices [2,3]. Most of the experimental studies of the proximity effect have focused on the analysis of threedimensional diffusive metals [4], whereas reports on twodimensional (2D) systems are scarce. Due to reduced screening, enhanced electron correlations, and localization at low temperatures, the proximity effect in 2D metals is expected to be drastically modified. However, not much is known about the interplay between superconducting correlations induced by proximity and electron correlations in disordered 2D metals. The central goal of this work is to shed new light on this fundamental issue.

So far, apart from notable exceptions [5-8], most of the reported experiments did not provide spatially resolved information on the proximity effect. This is mainly due to the difficulties on applying scanning tunneling microscopy (STM) or scanning tunneling spectroscopy (STS) techniques to ex situ elaborated (and thus, surfacecontaminated) mesoscopic systems. On the other hand, progress in the controlled growth of atomically clean superconducting materials under ultrahigh vacuum has opened the possibility to probe the proximity effect in in situ STM or STS experiments with high spatial and energy resolution. Very recently, Kim et al. [9] reported a STM or STS study in a system consisting of 5 monolayer (ML) thick superconducting $\mathrm{Pb}$ islands grown on $\mathrm{Si}(111)$ and an atomic overlayer of the striped incommensurate (SIC) phase of $\mathrm{Pb}$. Unfortunately, the analysis of the proximity effect in the SIC layer was performed at relatively high temperatures $(T=4.3 \mathrm{~K})$ and thus, the energy resolution was rather limited. More importantly, this experiment did not reveal any specific feature related to the low dimensionality of the SIC layer. Besides, SIC phase $\mathrm{Pb} / \mathrm{Si}(111)$ becomes superconducting below $T_{C}=1.8 \mathrm{~K}$ and thus, it is not an ideal system in which to study the proximity effect.

In this Letter we explore experimentally and theoretically the proximity effect in a highly diffusive atomically thin metal. The samples were grown in situ by the deposition at room temperature of a few atomic layers of $\mathrm{Pb}$ onto an atomically clean $7 \times 7$ reconstructed surface of undoped Si(111). The topographic STM images in Fig. 1(a) revealed the resulting $\mathrm{Pb}$ network consisting of atomically flat 7-13 ML thick single nanocrystals of $\mathrm{Pb}$, interconnected by a 1-2 ML thick wetting layer (WL) of $\mathrm{Pb}$ [10]. In Fig. 1(b) the experimental geometry is sketched; the regions of interest include the island edge and the neighboring part of the WL. The experiments were performed in ultrahigh vacuum $P \approx 5 \times 10^{-11}$ mbar and mechanically sharpened $\mathrm{Pt} / \mathrm{Ir}$ tips were used. In order to resolve fine spectroscopic features, the STM or STS experiments were conducted at $320 \mathrm{mK}$, i.e., at $T \approx T_{C} / 20$ [11]. Local tunneling conductance spectra $d I(V, \boldsymbol{r}) / d V$ were derived from the raw $I(V, r)$ data.

Our main finding is illustrated in Fig. 1(c) where we show the evolution of local $d I(V, Y) / d V$ spectra as a 

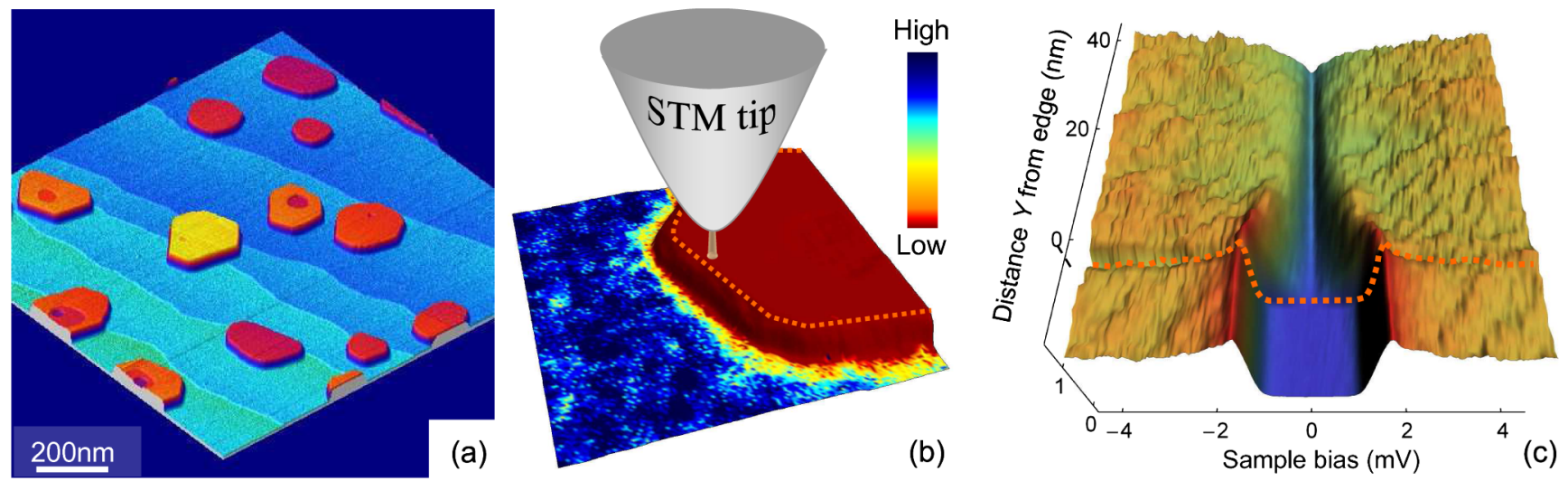

FIG. 1 (color online). (a) Topographic constant current $(I=0.1 \mathrm{nA}, V=25 \mathrm{mV})$ STM image: Pb islands (7-13 monolayers high) interconnected by the wetting layer cover single atomic terraces of underlying Si substrate. (b) Schematic representation of the STM or STS experiment with a real example of a color-coded STS tunneling conductance map $d I(V=0, \boldsymbol{r}) / d V$ superimposed onto $3 \mathrm{D}$ topographic STM image. The colors correspond to the conductance variations from $0 \mathrm{nS}$ ("Low") to 69 nS ("High"). The fully gaped spectra on the $\mathrm{Pb}$ island appear in homogeneous grey (red), the normal wetting layer in black (blue), and the proximity region in white (yellow). The dashed line indicates the position of the flat top edge of the $\mathrm{Pb}$ island. (c) Spatial variations of the local $d I(V) / d V$ spectra (the conductance varies from 0 to $100 \mathrm{nS}$ ) as a function of the distance from the island edge. The dashed line corresponds to the spectrum taken right at the island edge.

function of the distance $Y$ from the island edge. The superconducting state in the islands is characterized by a fully opened gap $d I(V=0) / d V=0$ [bottom curve in Fig. 1(c)], which is spatially homogeneous over the island area [red-colored in the STS map Fig. 1(b)]. On the WL side far from the islands, the zero-bias conductance is high, and the tunneling spectra in these regions exhibit a $V$-shaped dip at zero bias [upper curve in Fig. 1(c)], which from now on will be referred to as a zero-bias anomaly (ZBA). The absence of a clear gap is evidence of the intrinsic nonsuperconducting character of the WL [12]. As the STM tip approaches the island edge, the tunneling spectra evolve gradually from the nonsuperconducting ZBA to the superconducting gap shape thus evidencing the proximity effect on a scale of several nanometers (detailed conductance maps are presented in the Supplemental Material [13]).

Let us first discuss the origin of the ZBA in the tunneling spectra of the WL which is, taking into account its thickness and the absence of conduction electrons at low temperatures in the undoped Si substrate, an ideal case of a disordered 2D conductor. Indeed, among the reported $\mathrm{Pb}-\mathrm{Si}$ systems, the atomically disordered $\mathrm{Pb}$ WL [10] is characterized by an increased roughness in STM images $[\approx 0.06 \mathrm{~nm}$ in Fig. 2(b)] and lack of atomic ordering. This is at variance with the $\mathrm{Pb}$ islands which have smooth and perfectly ordered surfaces, see Fig. 2(a). In Fig. 2(c) we present a characteristic $d I / d V$ tunneling spectrum taken in the WL far enough from the islands, to avoid the proximity effect. The shape of this ZBA resembles the diffusive anomalies found in tunneling junctions between disordered conductors [14]. In those junctions, the suppressed tunneling conductance at low bias is attributed to the reduction of the electron tunneling DOS around the Fermi energy caused by the electron-electron interaction [14]. Similar anomalies appear in ultrasmall tunnel junctions due to dynamical Coulomb blockade (DCB) [15-18], i.e., due to the interaction of the tunneling electrons with the electromagnetic environment in which the junction is embedded [17].
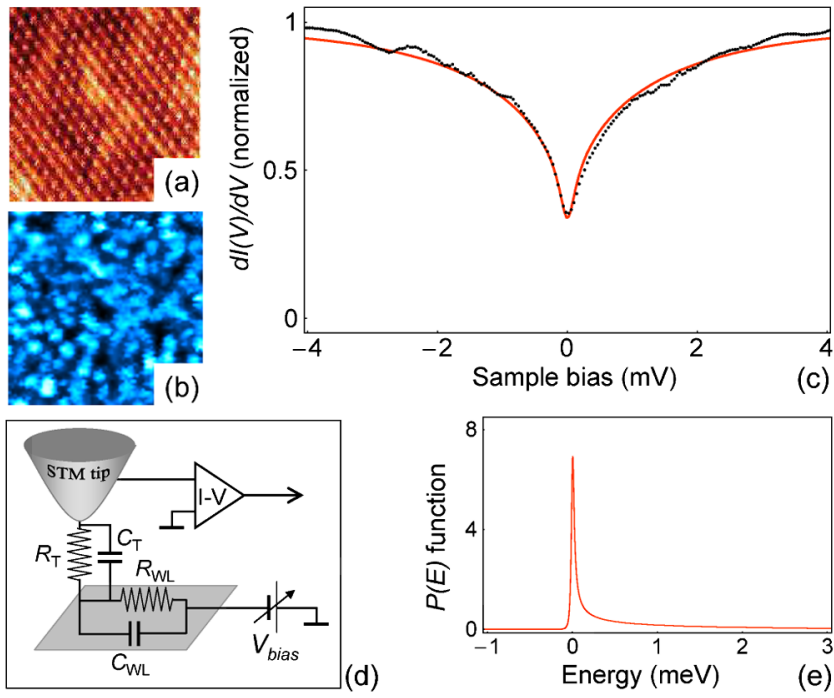

FIG. 2 (color online). (a) Topographic $5 \mathrm{~nm} \times 5 \mathrm{~nm}$ STM image (taken at $I=0.1 \mathrm{nA}, V=20 \mathrm{mV}$ ) showing the atomic pattern at the top of a Pb island. (b) Topographic $30 \mathrm{~nm} \times 30 \mathrm{~nm}$ STM image of the wetting layer. (c) Dotted line: characteristic conductance spectrum measured in the WL away from the $\mathrm{Pb}$ island (normalized to unity at STS set point $I=0.4 \mathrm{nA}$, $V=5 \mathrm{mV}$ ). The solid line corresponds to the fit obtained using the DCB theory described in the text. (d) Considered equivalent circuit of the experiment (see text for details). (e) The $P(E)$ function used to obtain the fit shown in panel (c). 
Indeed, these two phenomena have been shown to be two sides of the same coin [19-21]. In particular, Rollbühler and Grabert [21] have demonstrated that the tunneling into a disordered 2D conductor is formally equivalent to the DCB in an ultrasmall junction with an Ohmic environment. In view of this mapping, we shall use here the standard DCB theory detailed in Ref. [20] to describe the tunneling spectra in the normal part of the WL. Within this theory, the tunneling current is given by $I(V)=e\left[\Gamma_{\mathrm{WL} \rightarrow \mathrm{tip}}(V)-\right.$ $\left.\Gamma_{\text {tip } \rightarrow \mathrm{WL}}(V)\right]$, where the inelastic tunneling rates can be expressed as

$$
\begin{aligned}
\Gamma_{\mathrm{WL} \rightarrow \mathrm{tip}}(V)= & \frac{1}{e^{2} R_{\mathrm{T}}} \int_{-\infty}^{\infty} d E \int_{-\infty}^{\infty} d \epsilon n_{\mathrm{WL}}(E) \\
& \times f(E)[1-f(E-\epsilon+e V)] P(\epsilon)
\end{aligned}
$$

and $\Gamma_{\text {tip } \rightarrow \mathrm{WL}}(V)=\Gamma_{\mathrm{WL} \rightarrow \mathrm{tip}}(-V)$. Here, $R_{\mathrm{T}}$ is the tunneling resistance, $f(E)$ is the Fermi function, $n_{\mathrm{WL}}(E)$ is the normalized local DOS of the wetting layer, and $P(E)$ is the probability for an electron to emit the energy $E$ into the electromagnetic environment [20]. Notice that we have assumed that the tip has an energy-independent DOS. The $P(E)$ function is calculated using the total impedance $Z(\omega)=\left[i \omega C_{\mathrm{WL}}+1 / R_{\mathrm{WL}}\right]^{-1}$, where $C_{\mathrm{WL}}$ and $R_{\mathrm{WL}}$ are the effective capacitance and resistance of the WL, respectively, see Fig. 2(d). The STM junction capacitance, $C_{\mathrm{T}} \lesssim 1 \mathrm{aF}$ [18], was neglected here.

In Fig. 2(c) we show as a solid line the best fit of the experimental ZBA using the DCB theory just described. To obtain this fit by means of Eq. (1), both $R_{\mathrm{WL}}$ and $C_{\mathrm{WL}}$ were used as adjustable parameters, while the DOS of the WL was assumed to be the one of the noninteracting normal state, i.e., $n_{\mathrm{WL}}(E)=1$. The best fit for the experimental temperature was obtained with $C_{\mathrm{WL}}=80 \mathrm{aF}$ and $R_{\mathrm{WL}}=$ $3.22 \mathrm{k} \Omega$. The latter value is quite reasonable: It is close to yet lower than the quantum of resistance $h / e^{2}=25.8 \mathrm{k} \Omega$, as expected for a correlated $2 \mathrm{D}$ metal manifesting an Altshuler-Aronov ZBA [14]. The $R_{\mathrm{WL}}$ value is also consistent with previous in situ transport measurements [22]. The value of the capacitance $C_{\mathrm{WL}}$ is more difficult to interpret. It is about three orders of magnitude higher than the estimated self-capacitance $C_{\mathrm{ND}} \approx 5 \times 10^{-2} \mathrm{aF}$ of individual $\mathrm{Pb}$ nanodomains in Fig. 2(b), and almost two orders of magnitude larger than $C_{\mathrm{T}}$. This clearly indicates that the characteristic length scale involved in the charging effects is much larger than the typical nanometer size of a domain or of the tunneling junction and thus, is indeed related to the WL. Finally, the quality of the fit in Fig. 2(c) strongly supports that it is possible to render satisfactorily the tunneling data on the basis of the DCB theory using a minimalistic effective RC environment.

We now turn back to the spatial evolution of the tunneling spectra within the proximity region [23]. As the tip is moved away from the island and starts probing the WL, the tunneling conductance rapidly evolves, see Fig. 3(a).
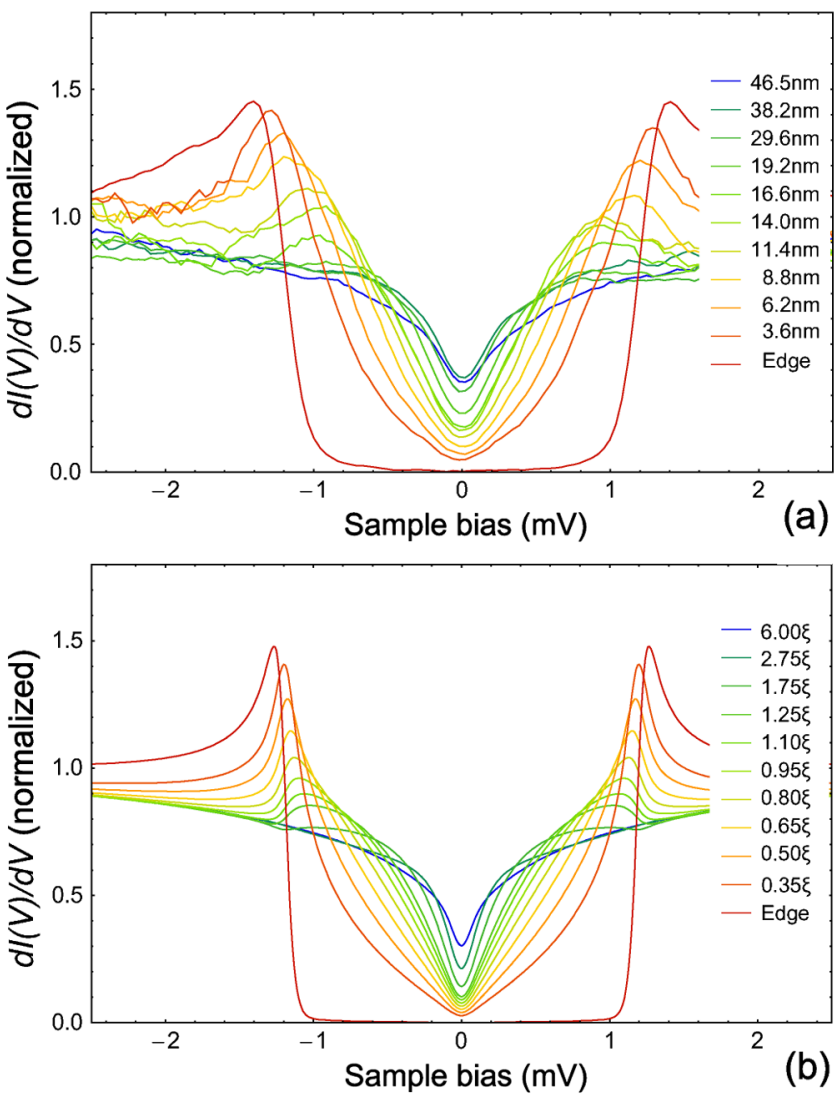

FIG. 3 (color online). (a) Evolution of measured local tunneling conductance spectra (normalized to unity at STS set point $I=0.4 \mathrm{nA}, V=5 \mathrm{mV}$ ) as a function of the distance from the island edge. (b) Computed spectra obtained with the combination of the Usadel model and the DCB theory (see text).

At short distances, the peak-to-peak separation smoothly reduces, the gap rapidly fills with quasiparticle states at the gap edges, while at zero-bias $d I(V=0, r) / d V$ remains low. At larger distances from the island, the peak-to-peak separation reduces further, the quasiparticle peaks rapidly smear out and reduce in height. Moreover, all of the tunneling characteristics inside the gap become more and more $V$ shaped, and the conductance at zero bias gradually increases. Finally, 40-50 $\mathrm{nm}$ away from the island the local $d I(V) / d V$ spectra become peakless and the ZBA of Fig. 2(c) is recovered (for 2D STS maps see Ref. [13]).

The theoretical description of the proximity effect in a correlated normal metal is challenging and, although considerable progress has been made in this direction [24], a suitable theory that can be directly applied to our hybrid system does not exist. In order to explain our experimental results, and inspired by Ref. [5], we shall use here a heuristic extension of the DCB theory discussed above. Our assumption is that the proximity effect can be described by computing the DOS of the WL in Eq. (1) with the help of the Usadel equations [25]. Let us recall that the Usadel theory has been very successful explaining the physics of conventional diffusive $S N$ junctions 
$[5,8,26]$. To be precise, we model our system by means of a $S N$ junction, where $N$ is an infinite normal wire and $S$ is an ideal BCS superconducting reservoir with a gap $\Delta$. We also assume that the $S N$ interface is perfectly transparent and we neglect the inverse proximity effect in the $S$ reservoir (see Ref. [13] for more details). In this model, the superconducting correlations penetrate in the normal metal a distance that depends on energy as $\sqrt{\hbar D / E}$, where $D$ is the diffusion constant in the metal.

To describe the results of Fig. 3(a), we use the $P(E)$ function obtained in the analysis of the normal state of the WL, see Fig. 2(e), which is now kept fixed. The superconducting gap is also fixed to $\Delta=1.2 \mathrm{meV}$ from the best fits of the spectra on the $\mathrm{Pb}$ island [27,28]; the distance to the $S N$ interface is measured in units of the length $\xi=$ $\sqrt{\hbar D / \Delta}$. Thus, the remaining adjustable parameter is the diffusion constant $D$ entering this expression, which must have the same value for all spectra. In Fig. 3(b) we present a set of numerically generated curves; the best match with experimental data is achieved with $\xi=15 \pm 3 \mathrm{~nm}$ resulting in $D \approx 4.1 \mathrm{~cm}^{2} / \mathrm{s}$. Notice that the computed spectra reproduce nicely the above discussed spatial evolution of the experimental tunneling data, including even very fine shape variations [29]. Moreover, the value of $D$ extracted here is in good agreement with the value inferred from in situ conductance measurements of these 1-2 monolayer thick WLs [22]. Let us emphasize that the diffusion constant in our case is more than one order of magnitude smaller than in conventional metals [5,8], which confirms that we are dealing with a highly disordered system (still away from the metal-insulator transition, $R_{\mathrm{WL}}<h / e^{2}$ ).

In conclusion, in a very low temperature STM or STS experiment we revealed the proximity effect between superconducting $\mathrm{Pb}$ islands and an ultimate disordered 2D metal - a nonsuperconducting $\mathrm{Pb}$ wetting layer grown on undoped $\mathrm{Si}(111)$. We found the tunneling spectra to evolve, on a scale of several nanometers, from a superconducting bulklike behavior inside the islands to a peculiar $V$-shaped zero-bias anomaly far away from them. We attributed this behavior to the interplay between disorder and electron correlations in the wetting layer. We developed a phenomenological combination of the DCB theory and the Usadel model to explain these observations. Our findings not only provide a novel insight into the physics of proximity effect, but they also pave the way for studying new aspects of this effect in a great variety of hybrid systems including in situ fabricated lateral SNS junctions in which the correlations play an important role.

We thank Hermann Grabert for useful discussions. This work was supported by grants from the University Pierre et Marie Curie (UPMC) "Emergence" and by CNRS Ph.D. Grant (L. S.-G.). J. C. C. and F. S. B. acknowledge financial support from the Spanish MICINN (Contracts No. FIS2011-28851-C02-01 and No. FIS2011-28851C02-02). *tristan.cren@upmc.fr

[1] P. G. de Gennes, Rev. Mod. Phys. 36, 225 (1964).

[2] J.-P. Cleuziou, W. Wernsdorfer, V. Bouchiat, T. Ondarçuhu, and M. Monthioux, Nat. Nanotechnol. 1, 53 (2006).

[3] F. Giazotto, J. T. Peltonen, M. Meschke, and J. P. Pekola, Nat. Phys. 6, 254 (2010).

[4] B. Pannetier and H. Courtois, J. Low Temp. Phys. 118, 599 (2000).

[5] S. Guéron, H. Pothier, N. O. Birge, D. Esteve, and M. H. Devoret, Phys. Rev. Lett. 77, 3025 (1996).

[6] N. Moussy, H. Courtois, and B. Pannetier, Europhys. Lett. 55, 861 (2001).

[7] W. Escoffier, C. Chapelier, N. Hadacek, and J.-C. Villegier, Phys. Rev. Lett. 93, 217005 (2004).

[8] H. le Sueur, P. Joyez, H. Pothier, C. Urbina, and D. Esteve, Phys. Rev. Lett. 100, 197002 (2008).

[9] J. Kim, V. Chua, G. A. Fiete, H. Nam, A. H. MacDonald, and C.-K. Shih, Nat. Phys. 8, 464 (2012).

[10] K. Budde, E. Abram, V. Yeh, and M. C. Tringides, Phys. Rev. B 61, R10602 (2000).

[11] C. Brun, I.-P. Hong, F. Patthey, I. Sklyadneva, R. Heid, P. Echenique, K. Bohnen, E. Chulkov, and W.-D. Schneider, Phys. Rev. Lett. 102, 207002 (2009).

[12] By applying an external magnetic field up to $6 \mathrm{~T}$ we additionally checked the absence of vortices in the WL and, more importantly, a general insensitivity of the ZBA spectra to the magnetic field.

[13] See Supplemental Material at http://link.aps.org/ supplemental/10.1103/PhysRevLett.110.157003 for a discussion of the shadow effect in the STM experiments, examples of 2D spatial maps of the proximity effect, and a detailed description of the theoretical model.

[14] B. L. Altshuler and A. G. Aronov, in Electron-Electron Interactions in Disordered Systems, edited by A.L. Efros and M. Pollak (Elsevier Science Publisher B. V., Amsterdam, 1985); P. A. Lee and T. V. Ramakrishnan, Rev. Mod. Phys. 57, 287 (1985).

[15] M.H. Devoret, D. Esteve, H. Grabert, G.-L. Ingold, H. Pothier, and C. Urbina, Phys. Rev. Lett. 64, 1824 (1990).

[16] S. M. Girvin, L. Glazman, M. Jonson, D. Penn, and M. Stiles, Phys. Rev. Lett. 64, 3183 (1990).

[17] Single Charge Tunneling, edited by H. Grabert and M. Devoret, NATO ASI, Ser. B Vol. 294 (Plenum, New York, 1992).

[18] C. Brun, K. H. Müller, I.-P. Hong, F. Patthey, C. Flindt, and W.-D. Schneider, Phys. Rev. Lett. 108, 126802 (2012).

[19] Yu. V. Nazarov, Zh. Eksp. Teor. Fiz. 95, 975 (1989) [Sov. Phys. JETP 68, 561 (1989)].

[20] G.-L. Ingold and Yu. V. Nazarov, in Single Charge Tunneling, edited by H. Grabert and M. Devoret, NATO ASI, Ser. B Vol. 294 (Plenum, New York, 1992), p. 21.

[21] J. Rollbühler and H. Grabert, Phys. Rev. Lett. 87, 126804 (2001).

[22] O. Pfennigstorf, A. Petkova, H.L. Guenter, and M. Henzler, Phys. Rev. B 65, 045412 (2002).

[23] Note that due to the finite size of the STM tip apex, the $\mathrm{WL}$ in the vicinity of the $\mathrm{Pb}$-island edge is not accessible by STS. Within this "shadow" region of a few nm the tip does not probe the WL but rather the upper edge of the island (see Ref. [13]). 
[24] Y. Oreg, P. W. Brouwer, B. D. Simons, and A. Altland, Phys. Rev. Lett. 82, 1269 (1999); M. A. Skvortsov, A. I. Larkin, and M. V. Feigel'man, Phys. Rev. B 63, 134507 (2001); A. G. Semenov, A. D. Zaikin, and L. S. Kuzmin, Phys. Rev. B 86, 144529 (2012).

[25] K. D. Usadel, Phys. Rev. Lett. 25, 507 (1970).

[26] W. Belzig, C. Bruder, and G. Schön, Phys. Rev. B 54, 9443 (1996).
[27] T. Nishio et al., Phys. Rev. Lett. 101, 167001 (2008).

[28] T. Cren, D. Fokin, F. Debontridder, V. Dubost, and D. Roditchev, Phys. Rev. Lett. 102, 127005 (2009); 107, 097202 (2011).

[29] Notice, however, that the agreement is not perfect, which shows the limitations of our simple model where a fixed $\mathrm{RC}$ environment was used to describe all the experimental curves of Fig. 3(a). 